\title{
Familial Mediterranean fever associated with optic neuritis, successfully treated with anti-interleukin 1 agents
}

\author{
Özge Başaran ${ }^{1}$, Sevim Kavuncu², Alev Güven ${ }^{2}$, Nermin Uncu ${ }^{1}$, Banu Acar-Çelikel ${ }^{1}$, \\ Nilgün Çakar ${ }^{1}$ \\ Departments of ${ }^{1}$ Pediatric Rheumatology and ${ }^{3}$ Pediatric Neurology, Ankara Child Health, Hematology, Oncology Training \\ and Research Hospital and 2Ankara Ulucanlar Eye Hospital, Ankara, Turkey. E-mail: ozgesalor@yahoo.com \\ Received: 14 July 2015, Revised: 21 January 2016, Accepted: 3 February 2016
}

SUMMARY: Başaran Ö, Kavuncu S, Güven A, Uncu N, Acar-Çelikel B, Çakar N. Familial Mediterranean fever associated with optic neuritis, successfully treated with anti-interleukin 1 agents. Turk J Pediatr 2016; 58: 327-330.

Familial Mediterranean fever (FMF) is an inherited periodic auto-inflammatory disease characterized by recurrent attacks of fever, synovitis and serositis. Ophthalmological manifestations of FMF are extremely rare.

Here we described a boy who has been followed-up for FMF and attended with a loss of vision during the course of the disease. He was diagnosed with optic neuritis. As the other etiologies were excluded his optic neuritis was attributed to the underlying auto inflammatory process. After pulse steroid therapy, his symptoms improved and a complete remission occurred. Afterwards he had two more optic neuritis attacks. Thereafter anti-interleukin 1 (IL-1) drugs were introduced and he did not develop further attacks of both optic neuritis and FMF.

This case presentation highlights the possible association between FMF and optic nerve involvement.

Key words: familial Mediterranean fever, optic neuritis, child, anti interleukin-1 agents.

Familial Mediterranean fever (FMF) is an auto-inflammatory disorder with recurrent attacks of fever and polyserositis lasting 1-4 days. FMF occurs frequently among Turkish, Jewish, Armenian, Arab and Italian population ${ }^{1}$. The responsible gene MEFV is located on chromosome $16 \mathrm{p}$ and produce a 781 amino acid protein called 'pyrin' or 'marenostrin'. Pyrin has a major role in caspase- 1 activation. This results in increased interleukin (IL) $1 \beta$ production which may induce the inflammatory phenotype of FMF patients. The first clinical episode starts during the childhood or adolescence ${ }^{2}$. FMF attacks occur due to excess inflammation of peritoneum, pleura, joints and skin, and sometimes in combination. Between episodes, patients are completely well. Diagnosis is based on the presence of short (12-72 hours), recurrent (3 or more) febrile attacks, with abdominal, chest, joint and skin involvement. Positive family history supports the diagnosis as well ${ }^{1,2}$. Colchicine is the main treatment of FMF. It prevents febrile episodes, manage FMF manifestations and it prevents the secondary systemic amyloidosis ${ }^{3}$. In addition, there are some reports of favorable response to anti-IL1 drugs in FMF refractory to colchicine therapy 4 . Ocular involvement of FMF is very rare and remains controversial. Also there have been only two patients in the literature having both FMF and optic neuritis $(\mathrm{ON})^{5}$. The patient we described here has been reported previously by Çakmaklı et al. ${ }^{6}$ in a clinical case series, discussing the $\mathrm{ON}$ in pediatric population. However, after the publication he had recurrent ON attacks which could not be prevented by steroid therapy and afterwards he had a good response with anti-IL-1 treatment. The aim of our describing this case here broadly is, to underline the possibility of FMF and recurrent $\mathrm{ON}$ association and to discuss the biological treatments in such patients.

\section{Case Report}

A 5-year-old boy with a diagnosis of FMF was admitted to our pediatric rheumatology 
clinic with a sudden loss of vision. His ophthalmological examination had revealed hemorrhage and swelling of optic disc. His visual acuity was 0.6 in right eye and counting fingers in left eye. Neurological examination did not reveal any abnormalities. In his previous history, he had episodic attacks of fever, abdominal pain and arthritis lasting for 3-4 days. His FMF DNA revealed M694V homozygotes. He had been followed up with the diagnosis of FMF for 2 years and had been receiving colchicine therapy with a dosage of $1 \mathrm{mg} /$ day. On his laboratory examination, blood cell count $6,800 / \mathrm{mm}^{3}$, platelets $418,000 /$ $\mathrm{mm}^{3}$, hemoglobin $12.5 \mathrm{~g} / \mathrm{dl}$, biochemical parameters including vitamin B12 were normal. Erythrocyte sedimentation rate (ESR) was raised at $80 \mathrm{~mm} /$ hour, C-reactive protein (CRP) was $4.31 \mathrm{mg} / \mathrm{ml}$ (range 0-0.8). Urinalysis was normal. Chest $\mathrm{X}$ ray was normal. Visual evoked potential (VEP) showed bilateral significant prolongation. Brain stem auditory evoked potential (BAEP) was normal. Cranial magnetic resonance imaging (MRI) was normal but on his orbital MRI there was bilateral thickness of optic nerves and ON. A diagnosis of retro bulbar neuritis was established and intra venous pulse methylprednisolone was given for 3 subsequent days $(30 \mathrm{mg} / \mathrm{kg} /$ day $)$. The treatment was continued with oral prednisolone ( $1 \mathrm{mg} / \mathrm{kg} /$ day). On his further investigations anti-nuclear antibody (ANA) and anti-ds DNA were negative, C3 and C4 levels were normal, anti-phospholipid immunoglobulin (Ig) $\mathrm{M}$ and $G$ were negative, Ig A,G and $M$ were within normal ranges. Serological testes for viral and bacterial investigations were negative. Cerebrospinal fluid (CSF) investigations including protein, glucose, viral markers, Ig $G$ index and oligoclonal band were normal. Human leucocyte antigen (HLA) was studied and displayed HLA B5. As he had no history of recurrent oral and genital ulcers, and his Pathergy test was negative, Behcet's disease was excluded. After four weeks from the steroid therapy optic disc swelling resolved and there was a progressive improvement of his visual acuity. His prednisolone dose was tapered. Three years later the patient was admitted with a blurry vision. On his ophthalmological examination, he had a pallor optic disc on the left eye. VEP also revealed no impulses on the left side. His orbital MRI showed active retro bulbar neuritis. Cranial MRI was normal $\mathrm{He}$ was started pulse methylprednisolone with a dosage of $30 \mathrm{mg} / \mathrm{kg} /$ day for 3 days and then continued with a high dose $(1 \mathrm{mg} / \mathrm{kg} /$ day $)$. Thereafter his visual acuity started improving. Steroid was tapered in a six months period. Two years later he was admitted again with an acute loss of vision. On his examination there was no pathology on the right eye but on the left he had retinal vascular thinning and optic disc swelling. VEP revealed no impulses on the left eye. Visual acuity was count fingers at 3 meters in right eye and 0.6 in left eye. Cranial MRI was normal. Orbital MRI was compatible with ON. Pulse methylprednisolone $(30 \mathrm{mg} / \mathrm{kg} /$ day, for 3 days) was started. After 2 months from the therapy his VEP revealed normal findings. His visual problems were improved and started tapering the steroid therapy. During his ON periods, he also had frequent FMF attacks (1-2 per month). Also his acute phase reactant levels were high. Although his colchicine dosage was increased up to $2 \mathrm{mg}$ /day, FMF attack frequency did not decrease. Anakinra (Kineret) was added to the therapy thereafter $(1 \mathrm{mg} / \mathrm{kg} /$ day, s.c. $)$. The dosage of anakinra was increased up to $3 \mathrm{mg} / \mathrm{kg} /$ day because of inadequate clinical response. Due to the ongoing worse clinical course anakinra was switched to canakinumab (Ilaris) therapy $(2 \mathrm{mg} / \mathrm{kg}$, monthly, s.c.). After the third dose of canakinumab, colchicine therapy was attempted to discontinue, but he had complaints of thoracic pain and slightly elevation of ESR and CRP. Therefore, he started colchicine again $(1 \mathrm{mg} / \mathrm{kg} /$ day). Two years after the initiation of anti IL1 treatment, ON and FMF attacks did not recur and inflammation markers were normal. His best-corrected visual acuity was 0.7 in right eye and 0.6 in left eye on his last ophthalmological visit.

\section{Discussion}

Ocular involvement in FMF patients has been reported previously. Those were uveitis, retinal ischemia, cataract, glaucoma and retinal detachment ${ }^{5,7,8}$. ON in FMF patient was reported in 1993 by Lossos et $\mathrm{al}^{4}$. They described two adult FMF patients (24 years old female and 28 year old male) who had developed ON during the course of FMF. They had only one ON episode and one of them received oral high dose prednisolone therapy 
and the other had no additional treatment other than colchicine. Both of them improved and their visual acuity returned to normal ${ }^{5}$.

Optic neuritis is an acquired inflammation of optic nerve and uncommon in pediatric population. Etiologies could be vascular, toxic/metabolic, neoplastic, infectious and noninfectious inflammatory 9 . Pathogenesis is due to an autoimmune reaction rather than the direct effect of infectious agents on optic disc. Pediatric ON may be the first feature of a systemic demyelination disease. In this setting, a work-up with cranial and orbital MRI, lumbar puncture and CSF studies including oligoclonal band and IgG index, and if needed further investigations for spinal MRI and neuromyelitis optica (NMO) IgG should be performed ${ }^{10,11}$. ON has been reported in the past with other immune mediated disorders like Behcet's disease, Sjogren's syndrome, Takayasu arteritis and systemic lupus erythematosus ${ }^{9,11-14}$. Those cases might support a probable relationship between autoimmune and autoinflamatory disorders and ON. Moreover, several studies have reported a possible link between NMO and systemic immune mediated diseases. NMO is an inflammatory disorder with $\mathrm{ON}$ and acute myelitis also affecting children ${ }^{11,15}$. ON may be the first presentation of a developing multiple sclerosis and NMO. Our patient had a normal cranial MRI, BAEP, and absence of oligoclonal bands in CSF analyses. On the other hand, during follow-up time (six years) in our clinic he did not develop further neurological features supporting any other neurological disease. Although it is not possible to completely exclude neurological disorders, we concluded that he developed isolated recurrent $\mathrm{ON}$ probably due to uncontrolled inflammation. In addition to this, he had started frequent FMF attacks and had high levels of inflammation markers during the optic neuritis episodes. Moreover, after initiation of anti IL-1 treatment, he had no more ON and FMF attacks and his acute phase reactant levels returned to normal ranges.

Colchicine has been reported before to induce the ocular and neurological complications due to vitamin B12 deficiency or its direct effects ${ }^{15}$. None of these complications were ON. The direct effect of colchicine to our patient could not be excluded. However, he had a normal serum vitamin B12 level and after his last ON attacks he continued receiving colchicine and he did not develop a new ON attack. Therefore we thought that his $\mathrm{ON}$ episodes were not a side effect of colchicine.

In summary, here we report a FMF patient who had developed recurrent attacks of ON concurrently with his FMF episodes. The significance of this case is not only that it is an extremely rare one but also it serves to make the clinician aware of the possibility of optic nerve involvement in FMF. It is also important to exclude the other etiological reasons of $\mathrm{ON}$ in pediatric population. After then in treatment modalities, it might be effective to introduce IL-1 targeting drugs in such cases to prevent the complications due to excessive inflammation.

\section{REFERENCES}

1. Yalçinkaya F, Ozen S, Ozçakar ZB, et al. A new set of criteria for the diagnosis of familial Mediterranean fever in childhood. Rheumatology 2009; 48: 395-398.

2. Livneh A, Langevitz P, Zemer D, et al. Criteria for the diagnosis of familial Mediterranean fever. Arthritis Rheum 1997; 40: 1879-1885.

3. Kallinich T, Haffner D, Niehues T, et al. Colchicine use in children and adolescents with familial Mediterranean fever: literature review and consensus statement. Pediatrics 2007; 119: e474-e483.

4. Dinarello CA. Interleukin-1beta and the autoinflammatory diseases. N Engl J Med 2009; 360: 2467-2470.

5. Lossos A, Eliashiv S, Ben-Chetrit E, Reches A. Optic neuritis associated with familial Mediterranean fever. J Clin Neuroophthalmol 1993; 13: 141-143.

6. Cakmakli G, Kurne A, Guven A, et al. Childhood optic neuritis: the pediatric neurologist's perspective. Eur J Paediatr Neurol 2009; 13: 452-457.

7. Hirsh A, Huna R, Ashkenazi I, Bartov E, Blumenthal $M$. Recurrent bilateral panuveitis and rhegmatogenous retinal detachment in a patient with familial Mediterranean fever. Am J Ophthalmol 1990; 110: 702-703.

8. Akman-Demir G, Gul A, Gurol E, et al. Inflammatory/ demyelinating central nervous system involvement in familial Mediterranean fever (FMF): coincidence or association? J Neurol 2006; 253: 928-934.

9. El-Dairi MA, Ghasia F, Bhatti MT. Pediatric optic neuritis. Int Ophthalmol Clin 2012; 52: 29-49.

10. Costello F. Inflammatory optic neuropathies. Continuum (Minneap Minn) 2014; 20: 816-837.

11. Collinge JE, Sprunger DT. Update in pediatric optic neuritis. Curr Opin Ophthalmol 2013; 24: 448-452.

12. Tang WQ, Wei SH. Primary Sjögren's syndrome related optic neuritis. Int J Ophthalmol 2013; 6: 888-891. 
13. Pyo JY, Park JS, Song CH, Lee SW, Park YB, Lee SK. Takayasu arteritis associated with ulcerative colitis and optic neuritis: first case in Korea. Korean J Intern Med 2013; 28: 491-496.

14. Frigui M, Frikha F, Sellemi D, Chouayakh F, Feki J, Bahloul Z. Optic neuropathy as a presenting feature of systemic lupus erythematosus: two case reports and literature review. Lupus 2011; 20: 1214-1218.
15. Wingerchuk DM, Weinshenker BG. The emerging relationship between neuromyelitis optica and systemic rheumatologic autoimmune disease. Mult Scler 2012; 18: 5-10.

16. Palopoli JJ, Waxman J. Colchicine neuropathy or vitamin B12 deficiency neuropathy? N Engl J Med 1987; 317: 1290-1291. 